\title{
A Note on Usage
}

In this book, I've made a practice of adhering to word usage that was conventional during the period I'm examining. For that reason, I've chosen to use the word "Negro" throughout, instead of "African American." I trust this will give no offense. 
